\title{
Correction to "uniqueness for the harmonic map flow from surfaces to general targets"
}

(Comment. Math. Helvetici 70 (1995) 310-338)

\section{A. Freire}

The calculation leading to estimate (3.8) in the paper is incorrect. Thus the construction of adapted p-frames described in the paper is not valid, and the existence of such frames adapted to a general time-dependent map (Theorem 3.1) remains in doubt. What is possible to obtain are tangent frames which are 'optimal' in a certain sense, but only for each fixed time. As described below, this turns out to be sufficient to prove the main theorem 1.1 as stated in the paper. For convenience of the reader, we recall its statement. We consider weak solutions of the heat flow for harmonic maps with initial data $u_{0} \in H^{1}(M ; N)$, where $N$ is a $\mathrm{k}$-dimensional compact embedded submanifold of $\mathbb{R}^{p}$, with the induced Riemannian metric. Define:

$$
V^{T}=H^{1}(M \times[0, T] ; N) \cap L^{\infty}\left([0, T] ; H^{1}(M ; N)\right) \cap L^{2}\left([0, T] ; H^{2}(M, N)\right) .
$$

By work of M. Struwe [1], a solution $v \in V^{T}$ exists for sufficiently small $T>0$ (depending on $u_{0}$ ), and may be continued to a global weak solution with finite singular set in $M \times(0, \infty)$. We refer to $v$ as the 'almost regular solution'.

THEOREM 1.1. Let $u \in H^{1}(M \times[0, T] ; N)$ be a weak solution of the harmonic map flow with initial conditions $u_{0} \in H^{1}(M, N)$. Assume $E_{u}(t) \leq E_{u_{0}}$ a.e. in $I=[0, T]$. Then there exists $T^{\prime} \in(0, T)$ such that $u \in V^{T^{\prime}}$.

The proof proceeds in two steps. First, using a modified 'optimal frames' construction for each constant time (Lemma A) and a lemma of Hélein [16] (Lemma D), we obtain a regularity result for each $u(t)$ (Lemma B). Then we conclude the proof by the same 'perturbation argument' used in the paper (Lemma 2.2).

Theorems, lemmas, equations and references in the paper are referred to here by the same number. The notation is the same, with the following additional conventions. $\mathbb{F}^{k, p}$ denotes the space of orthonormal $\mathrm{k}$-frames in $\mathbb{R}^{p}$. In the notation of frames $e_{i}$ and connection forms $\omega_{i j}$ the indices $i, j$ are usually omitted for brevity. 
$c>0$ denotes a generic positive constant whose value may depend on $M, N$, a given smooth tangent orthonormal frame $\bar{e}$ on $N$, and on $u_{0}$.

For simplicity the argument below is given in the case of surfaces without boundary.

The statement and proof of Theorem 3.1 (existence of adapted frames) should be changed as follows.

Let $\bar{e}=\left(\bar{e}_{i}\right)_{i=1}^{k}, \bar{e} \in L^{\infty}\left(I ; H^{1}\left(M ; \mathbb{F}^{k, p}\right)\right)$, be the 'background' adapted frame obtained by composing a smooth orthonormal frame tangent to $N$ with $u(x, t)$. Since $u(t) \rightarrow u_{0}$ strongly in $H^{1}(M ; N)$ as $t \rightarrow 0$ (by the energy bound hypothesized in the theorem), we also have $\bar{e}(t) \rightarrow \bar{e}(0)$ strongly in $H^{1}\left(M ; \mathbb{F}^{k, p}\right)$. Let $\epsilon>0$ be given. We define $T_{\epsilon} \in(0, T)$ as follows. Since $\operatorname{dim}(M)=2$, we may write:

$$
\bar{e}(0)=\bar{e}^{-1}(0)+\bar{e}^{2}(0),
$$

where $\bar{e}^{-2}(0) \in C^{\infty}\left(M ; \mathbb{F}^{k, p}\right)$ and $\bar{e}^{-1}(0) \in\left(H^{1} \cap L^{\infty}\right)\left(M ; \mathbb{R}^{p}\right)$ satisfies $\left\|\bar{e}^{-1}(0)\right\|_{H^{1}}<\epsilon / 2$. Fix $T_{\epsilon}>0$ such that $\|\bar{e}(t)-\bar{e}(0)\|_{H^{1}}<\epsilon / 2$ for all $t \in\left[0, T_{\epsilon}\right]$ such that $\bar{e}(t) \in H^{1}$. Then, setting $\bar{e}^{-1}(t)=\bar{e}(t)-\vec{e}^{2}(0)$, we have:

$$
\left\|\bar{e}^{-1}(t)\right\|_{H^{1}}<\epsilon
$$

for all $t \in\left[0, T_{\epsilon}\right]$ such that $\bar{e}(t) \in H^{1}$.

The result that replaces theorem 3.1 is:

LEMMA A. Let $\epsilon>0$ be given, and choose $T_{\epsilon}>0$ as above. Fix $t \in\left[0, T_{\epsilon}\right]$ such that $u(t) \in H^{1}(M ; N)$. We may find a tangent orthonormal frame $e(t) \in H^{1}\left(M ; \mathbb{F}^{k, p}\right)$ adapted to $u(t)$, whose connection 1-form $\omega_{i j}=\left\langle d e_{i}, e_{j}\right\rangle$ admits the decomposition:

$$
\omega(t)=\omega^{1}(t)+\omega^{2}(t)
$$

with $\delta \omega^{1}(t)=0,\left\|\omega^{1}(t)\right\|_{2}<\epsilon$ and $\omega^{2}(t) \in L^{\infty} \Omega^{1}(M) \otimes s o(k)$, with norm bounded by a constant independent of $t$.

Proof. Let $\bar{\omega}_{i j}^{1}(t)=\left\{\left\langle d \bar{e}_{i}^{1}(t), \bar{e}_{j}(t)\right\rangle\right\}, \bar{\omega}_{i j}^{2}(t)=\left\{\left\langle d \bar{e}_{i}^{2}(0), \bar{e}_{j}(t)\right\rangle\right\}$. Consider a 'gauge transformation' $g \in H^{1}$; $S O(k)$ ) which minimizes the functional $F(g)$ given by:

$$
F(g)=\int_{M}\left|d g g^{t}+g \bar{\omega}^{1}(t) g^{t}\right|^{2} d x,
$$

where the superscript ' $t$ ' denotes 'transpose'. A minimizer clearly exists. The Euler-Lagrange equation for $F$ is: 


$$
\delta \omega^{1}(t)=0
$$

where: $\omega^{1}(t)=d g g^{t}+g \omega^{1}(t) g^{t}$. Since $g$ is a minimizer,

$$
\left\|\omega^{1}(t)\right\|_{2} \leq\left\|\bar{\omega}^{1}(t)\right\|_{2}<\epsilon .
$$

Let $e_{i}(t)=\Sigma_{j} g_{i j} \bar{e}_{j}(t)$. The connection 1-forms $\omega$ of $e(t)=\left(e_{i}\right)(t)$ may be decomposed as:

$$
\omega(t)=d g g^{t}+g \bar{\omega}(t) g^{t}=\omega^{1}(t)+\omega^{2}(t),
$$

where $\omega^{2}(t)=g \bar{\omega}^{2}(t) g^{t}$. Since $\left\|\bar{\omega}_{i j}^{2}(t)\right\|_{\infty} \leq\left\|d \bar{e}_{i}^{2}(0)\right\|_{\infty} \leq c$, this concludes the proof.

Remark $A . \omega^{1}$ is not the connection 1 -form of a frame. However, defining $e^{1}=g \bar{e}^{-1}$, we have $\left(\right.$ since $\left.\langle\bar{e}, e\rangle=g^{t}\right)$ :

$$
\begin{aligned}
\left\langle d e^{1}, e\right\rangle & =d g\left\langle\bar{e}^{1}, e\right\rangle+g\left\langle d \bar{e}^{1}, e\right\rangle \\
& =d g g^{t}-d g\left\langle\bar{e}^{2}, e\right\rangle+g\left\langle d \bar{e}^{1}, \bar{e}\right\rangle\langle\bar{e}, e\rangle \\
& =d g g^{t}+g \bar{\omega}^{1} g^{t}-d g\left\langle\bar{e}^{2}, e\right\rangle ;
\end{aligned}
$$

so $\omega^{1}=\left\{\left\langle d e^{\mathrm{i}}, e\right\rangle+d g\left\langle\vec{e}^{2}, e\right\rangle\right\}$. (In this calculation we have denoted, for instance, by $\left\langle\bar{e}^{-1}, e\right\rangle$ the matrix with $i j$-th entry $\left\langle\bar{e}_{i}^{1}, e_{j}\right\rangle$.)

Lemma $\mathrm{A}$ gives no control on the connection 1-forms $\omega_{i a}\left(e_{i}\right.$ tangential, $e_{a}$ normal). Thus the proof of theorem 1.1 must be modified. This is accomplished by using a lemma of Hélein, which allows us to give a simpler proof than the argument attempted in the paper. The main step is the following lemma.

LEMMA B. Consider a solution $u: M \times I \rightarrow N$ of the harmonic map flow to $N$ satisfying the assumptions of theorem 1.1. There exists $\epsilon_{1}>0$ with the following property. Define $T_{\epsilon_{1}}>0$ as in the paragraph preceding Lemma $A$. Let $t \in\left(0, T_{\epsilon_{1}}\right]$ satisfy $u_{t}(t) \in L^{2}\left(M ; \mathbb{R}^{p}\right), u(t) \in H^{1}(M, N)$. Then $d u(t) \in L^{4} \Omega_{M}^{1}$ and

$$
\|d u(t)\|_{4} \leq c\left(1+\left\|u_{t}(t)\right\|_{2}\right)
$$

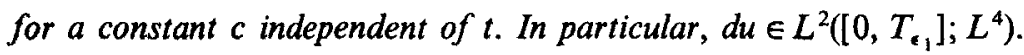

The conclusion of lemma $\mathrm{B}$ is 'higher regularity of $u$ in space directions'. It is then easy to conclude $u$ is in the class $V^{T \prime}$ (for some $T^{\prime}<T$ ) as claimed in theorem 1.1 , by means of a 'perturbation argument' based on linear parabolic theory. This 
is accomplished in the following lemma (which is essentially a restatement of Lemma 2.2 in the paper).

Consider the general non-homogeneous linear parabolic system of the form:

$$
\begin{cases}\Phi_{t}-\Delta \Phi=-(d \Phi \cdot \omega) e+f(x, t) & \text { on } M \times I \\ \Phi(., 0)=0 & \text { in } M\end{cases}
$$

LEMMA C. There exists $\epsilon_{2}>0$ (depending on $M$ and $T$ ) with the following property. Let $I^{\prime}=\left[0, T^{\prime}\right]$, where $T^{\prime} \in(0, T]$ is arbitrary. Let $f \in L^{4}\left(I^{\prime}, L^{4 / 3}\right)$, $\omega \in L^{\infty}\left(I^{\prime}, L^{2} \Omega^{1}\right)$ and $e \in L^{\infty}\left(M \times I^{\prime}\right)$, $\|e\|_{\infty} \leq 1$. Assume $\|\omega\|_{L^{\infty}\left(r, L^{2}\right)}<\epsilon_{2}$. Let $\Phi$ be a solution of $(L)$ in $\left(H^{1} \cap L^{\infty}\right)\left(M \times I^{\prime}\right)$, such that $\|\nabla \Phi\|_{L^{4}(M)} \in L^{2}\left(I^{\prime}\right)$. Then $\Phi \in L^{4}\left(I^{\prime}, W^{2,4 / 3}\right)$. (Note: $\epsilon_{2}$ is independent of $T^{\prime \prime}$.)

Proof. The first part of the proof of Lemma 2.2 shows that, for each $p \in(1,4]$, there exists $\epsilon(p)>0$ such that system $(L)$ has unique solutions in $L_{0}^{p}\left(I^{\prime}, W^{2,4 / 3}\right)$. In particular one may find $\epsilon_{2}>0$ such that $(L)$ has a unique solution $\Phi_{1} \in$ $L_{0}^{4}\left(I^{\prime}, W^{2,4 / 3}\right)$, and also a unique solution in $L_{0}^{2}\left(I^{\prime}, W^{2,4 / 3}\right)$. The latter must coincide with the given $\Phi$, since $\|\nabla \Phi\|_{L^{4}(M)} \in L^{2}\left(I^{\prime}\right)$ implies (by linear theory, Theorem 2.1) $\Phi \in L_{0}^{2}\left(I^{\prime}, W^{2,4 / 3}\right)$. Since $L_{0}^{4}\left(I^{\prime}, W^{2,4 / 3}\right) \hookrightarrow L_{0}^{2}\left(I^{\prime}, W^{2,4 / 3}\right)$, uniqueness in $L_{0}^{2}\left(I^{\prime}, W^{2,4 / 3}\right)$ implies $\Phi=\Phi_{1}$.

Proof of Theorem 1.1. Using the background frame $\left\{\vec{e}_{r}\right\}_{r=1}^{p}$, we write the equation for $u$ in the form (2.4a):

$$
u_{t}-\Delta u=-\sum_{i, a}\left\langle d u \cdot \bar{\omega}_{i a}, \bar{e}_{i}\right\rangle \bar{e}_{a}
$$

Let $\epsilon_{2}$ be given by lemma C. Choose $T^{\prime}<\min \left\{T_{\epsilon_{1}}, T_{\epsilon_{2}}\right\}$. Then for $t \in\left[0, T^{\prime}\right]$ the following decomposition holds:

$$
\bar{\omega}_{i a}(t)=\bar{\omega}_{i a}^{1}(t)+\bar{\omega}_{i a}^{2}(t)
$$

where $\left\|\bar{\omega}_{i a}^{1}(t)\right\|_{2}<\epsilon_{1}$ and $\bar{\omega}_{i a}^{2} \in L^{\infty}\left(M \times\left[0, T^{\prime}\right]\right)$. Letting $v: M \times\left[0, T_{0}\right] \rightarrow \mathbb{R}^{p}$ be the solution of the linear heat equation with initial data $u_{0}$, we obtain for $w=u-v$ :

$$
\left\{\begin{array}{l}
w_{t}-\Delta w=-\sum_{i, a}\left\langle d w \cdot \bar{\omega}_{i a}^{1}, \bar{e}_{i}\right\rangle \bar{e}_{a}+f(x, t), \\
w(., 0)=0
\end{array}\right.
$$

where:

$$
f(x, t)=\left\langle d v \cdot \bar{\omega}_{l a}^{1}, \bar{e}_{i}\right\rangle \bar{e}_{a}+\left\langle d w \cdot \bar{\omega}_{l a}^{2}, \bar{e}_{t}\right\rangle \bar{e}_{a} \in L^{4}\left(\left[0, T^{\prime}\right], L^{4 / 3}\right) .
$$


Indeed we have (as in subsection 2.3):

$$
\begin{aligned}
\left\|d v \cdot \bar{\omega}_{i a}^{1}\right\|_{L^{4 / 3}\left(M_{t}\right)} & \leq c\|d v\|_{L^{4}\left(M_{t}\right)}\left\|\bar{\omega}_{i a}^{1}\right\|_{L^{2}\left(M_{t}\right)} \\
& \leq c\|d v\|_{L^{2}\left(M_{t}\right)}^{1 / 2}\|v\|_{H^{2}\left(M_{i}\right)} \epsilon_{1} \\
\left\|d w \cdot \bar{\omega}_{i a}^{2}\right\|_{L^{4 / 3}\left(M_{t}\right)} & \leq c\|d w\|_{L^{2}\left(M_{t}\right)}\left\|\bar{\omega}_{i a}^{2}\right\|_{L^{4}\left(M_{t}\right)},
\end{aligned}
$$

which implies:

$$
\|f\|_{L^{4}\left(I, L^{4 / 3}\right)}^{4} \leq c \epsilon_{1}^{4}\|v\|_{L^{2}\left(I, H^{2}\right)}^{2}+c T^{\prime}\|d w\|_{L^{\infty}\left(I, L^{2}\right)}^{4}\left\|\bar{\omega}_{i a}^{2}\right\|_{L^{\infty}(M \times \Gamma)}^{4}
$$

By lemma $\mathrm{B}, d w \in L^{2}\left(\left[0, T^{\prime}\right], L^{4}\right)$. Thus we may apply lemma $\mathrm{C}$ to $w$ and conclude $w \in L_{0}^{4}\left(\left[0, T^{\prime}\right], W^{2,4 / 3}\right)$, hence $u \in L^{4}\left(\left[0, T^{\prime}\right], W^{2,4 / 3}\right)$. From the embedding $W^{2,4 / 3} \hookrightarrow W^{1,4}$ and the equation of the flow this implies $u \in L^{2}\left(\left[0, T^{\prime}\right], H^{2}\right)$, as claimed in the theorem.

Proof of lemma $B$. (1) Let $\epsilon_{1}=c_{1} \epsilon_{0}$, where $c_{1}$ is the constant defined in paragraph (4) below and $\epsilon_{0}$ is given by Lemma $\mathrm{D}$ below. Let $T_{\epsilon_{1}}>0$ be chosen as in Lemma A above. Fix $t \in\left(0, T_{\epsilon_{1}}\right]$ such that $u_{t}(t) \in L^{2}$, and let $e(t)=\left(e_{i}\right)(t)$ be the adapted frame given by Lemma $A$, whose connection 1 -forms decompose as in its statement: $\omega(t)=\omega^{1}(t)+\omega^{2}(t)$. Since $\delta \omega^{1}(t)=0$, we have the Hodge decomposition:

$$
\omega^{1}(t)=\delta B(t)+H(t)
$$

where $B(t) \in H^{1} \Omega_{M}^{2} \otimes s o(k)$ satisfies $\int_{M} B(t)=0$ and:

$$
\|B\|_{H^{1}} \leq c\left\|\omega^{1}\right\|_{2}<c \epsilon
$$

and $H(t)$ is a harmonic 1 -form on $M$.

(2) In order to use Hélein's lemma we must localize the problem. Cover $M$ by open sets $\left\{U_{\alpha}\right\}_{\alpha=1}^{N}$, such that:

(i) there exist conformal coordinate charts $\varphi_{\alpha}: D_{2} \rightarrow M, \varphi_{\alpha}\left(D_{2}\right)=U_{\alpha}$;

(ii) $M \subset \bigcup_{i=1}^{N} V_{\alpha}, V_{\alpha}=\varphi_{\alpha}\left(D_{1 / 2}\right)$.

We denote by $D_{r}$ the open disk with radius $r$ centered at the origin in $C\left(D=D_{1}\right)$, endowed with the metric $d s^{2}=\lambda^{2}|d z|^{2}$, pulled back from $M$ via the local charts $\varphi_{\alpha}$. Fix an index $\alpha$ for the remainder of the proof, and for simplicity of notation identify the maps $u, e_{i}$, etc. with their pullbacks to $D_{2}$ under $\varphi_{\alpha}$. 
Let

$$
\begin{aligned}
\alpha_{i}(t) & =\left\langle u_{z}, e_{i}(t)\right\rangle=\frac{1}{2}\left\langle u_{x}-i u_{y}, e_{i}(t)\right\rangle \in L^{2}\left(D_{2}\right), \\
a_{i j} & =\left\langle\left(e_{i}(t)\right)_{z}, e_{j}(t)\right\rangle \\
& =\frac{1}{2}\left[\left(\omega_{i j}\right)\left(\partial_{x}\right)+i \omega_{i j}\left(\hat{\partial}_{y}\right)\right] \in L^{2}\left(D_{2}\right)
\end{aligned}
$$

(with $L^{2}$ norm independent of $t$ in both cases). Then:

$$
\begin{aligned}
\left(\alpha_{i}\right)_{\bar{z}} & =\left\langle u_{z \bar{z}}, e_{i}\right\rangle+\left\langle u_{z},\left(e_{i}\right)_{\bar{z}}\right\rangle \\
& =\frac{1}{4}\left\langle\lambda^{2} \Delta u, e_{i}\right\rangle+\sum_{j} \alpha_{j} a_{i j},
\end{aligned}
$$

( 4 denotes the Laplacian in the Euclidean metric) so:

$$
\left(\alpha_{i}\right)_{\bar{z}}+\sum_{j} a_{j i} \alpha_{j}=\frac{\lambda^{2}}{4}\left\langle u_{t}, e_{i}\right\rangle
$$

(3) By remark A above, we have:

$$
\Delta B=d \omega^{1}=\left\{d e^{1} \wedge d e-d g \wedge d\left\langle\bar{e}_{2}, e\right\rangle\right\}_{-} \in \mathscr{H}_{l o c}^{1}\left(D_{2}\right) .
$$

( $\mathscr{H}_{l o c}^{1}$ denotes a local Hardy space, as in [16]). Therefore $B \in W_{\text {loc }}^{2,1}\left(D_{2}\right) \subset C^{0}\left(D_{2}\right)$ and:

$$
\|B\|_{W^{2,1}\left(D_{1}\right)} \leq c\left(\left\|d e^{1}\right\|_{2}\|d e\|_{2}+\|d g\|_{2}\|e\|_{H^{1}}\right) .
$$

We also have the estimates (in $D_{1}$ ):

$$
\begin{gathered}
\|d g(t)\|_{2}=\left\|\omega^{1}(t) g(t)-g(t) \bar{\omega}^{1}(t)\right\|_{2} \leq 2\left\|\bar{\omega}^{1}(t)\right\|_{2}<2 \epsilon, \\
\left\|d e^{1}(t)\right\|_{2}=\left\|(d g) \bar{e}^{-1}+g d \bar{e}^{1}\right\|_{2} \leq c\left(\|d g\|_{2}+\left\|\bar{e}^{1}\right\|_{H^{1}}\right)<c \epsilon,
\end{gathered}
$$

so we conclude $\|B\|_{W^{2.1}\left(D_{1}\right)} \leq c \epsilon$.

(4) Corresponding to the decomposition:

$$
\omega=\delta B+\omega^{2}+H,
$$


we set:

$$
\omega\left(\partial_{\bar{z}}\right)=a(t)=a^{1}(t)+a^{2}(t)+a^{3}(t)
$$

where (and here we define $c_{1}$ ):

$$
\begin{aligned}
a^{1}(t) & =\delta B\left(\partial_{\bar{z}}\right) \in W^{1,1}(D), \quad\left\|a^{1}\right\|_{W^{1,1}}<c_{1} \epsilon, \\
a^{2}(t) & =\omega^{2}\left(\partial_{\bar{z}}\right) \in L^{\infty}(D), \\
a^{3}(t) & =H(t)\left(\partial_{\bar{z}}\right) \in L^{\infty}(D),
\end{aligned}
$$

with $L^{\infty}$ norm bounded independently of $t$ in the last two cases.

We may rewrite (1) in the form:

(2) $\quad\left(\alpha_{i}\right)_{\bar{z}}+\sum_{j} a_{j i}^{1} \alpha_{j}=f_{i}$

where:

$$
f_{i}=\frac{\lambda^{2}}{4}\left\langle u_{t}(t), e_{i}(t)\right\rangle+\sum_{j}\left(a_{i j}^{2}+a_{i j}^{3}\right) \alpha_{j}(t) \in L^{2}(D)
$$

with:

$$
\begin{aligned}
\left\|f_{i}(t)\right\|_{L^{2}(D)} & \leq c\left(\left\|u_{t}(t)\right\|_{L^{2}(D)}+\left\|\left\langle d u, e_{i}(t)\right\rangle\right\|_{L^{2}(D)}\right) \\
& \leq c\left(1+\left\|u_{t}(t)\right\|_{L^{2}(D)}\right) .
\end{aligned}
$$

(5) LEMMA D. (Hélein [16]) There exist constants $\epsilon_{0}>0, c_{0}>0$ such that if $a_{i j} \in W^{1,1}(D),\left\|a_{i j}\right\|_{W^{1,1}}<\epsilon_{0}$, one may find solutions $\beta^{k} \in L^{\infty}\left(D, \mathbb{C}^{n}\right), k=1, \ldots, n$ of the system:

$$
\left(\beta_{i}\right)_{\bar{z}}=\sum_{j} a_{i j} \beta_{j}, \quad \beta^{k}=\left(\beta_{i}^{k}\right)_{i=1}^{n}
$$

and a map $M=\left(m_{i j}\right) \in L^{\infty}(\mathrm{D}, G L(n, \mathbb{C}))$ such that:

$$
\|M\|_{L^{\infty}}+\left\|M^{-1}\right\|_{L^{\infty}} \leq c_{0} \quad\left\|\beta^{j}-b^{j}\right\|_{L^{\infty}} \leq c_{0}
$$

and $b^{k}=\sum_{j=1}^{n} m_{k j} \beta^{j}$, where $b^{1}, \ldots, b^{n}$ is the standard basis of $\mathbb{C}^{n}$. 
(6) We have (as in [16]):

$\alpha_{j}=\sum_{i} \delta_{j i} \alpha_{i}=\sum_{k, i} m_{j k} \beta_{i}^{k} \alpha_{i}=\sum_{k} m_{j k} \gamma^{k}$,

where $\gamma^{k}=\Sigma_{i} \beta_{i}^{k} \alpha_{i}$ and (2) implies:

$$
\gamma_{z}^{k}=\sum_{i}\left[\left(\beta_{i}^{k}\right)_{\bar{z}} \alpha_{i}+\beta_{i}^{k}\left(\alpha_{i}\right)_{\bar{z}}\right]
$$

(4) $\quad=\sum_{i} \beta_{i}^{k} f_{i} \in L^{2}(D)$

By elliptic regularity for $\bar{\partial}$, this implies:

$$
\gamma^{k}(t) \in H^{1}\left(D_{1 / 2}\right),
$$

and hence $\gamma^{k}(t)$ (and therefore $\alpha_{j}$ ) is in $L^{p}\left(D_{1 / 2}\right)$ for each $1<p<\infty$. In particular, this clearly implies $d u(t) \in L^{4}\left(V_{\alpha}\right)$ ) for each $\alpha$. Thus $d u \in L^{4} \Omega_{M}^{1}$. The estimate claimed in the lemma follows from (3) and (4).

University of Tennessee

Dept of Mathematics

Knoxville, Tennessee 37996-1300

USA 